\title{
Fluorescence Spectroscopic and Thermodynamic Studies of Chlorophyll Containing Monolayers and Vesicles. Part I: Mixed Monolayers of Pheophytin A and Lecithin
}

\author{
H. Heithier, H.-J. Galla, and H. Möhwald \\ Experimentelle Physik III, Universität Ulm \\ Z. Naturforsch. 33 c, 382-391 (1978) ; received March 15/April 3, 1978 \\ Monolayers, Chlorophyll, Energy Transfer, Fluorescence Spectroscopy, Phase Transitions \\ Mixed monolayers of pheophytin a and $\alpha$-L-dimyristoyl lecithin (DML) are investigated on the \\ water surface. The studies gain their special value from the simultaneous measurement of surface \\ pressure and fluorescence intensity as a function of the covered area per molecule. \\ A phase separation in the liquid state of the monolayer is established. Phase 1 exists almost \\ exclusively of pheophytin molecules. Phase 2 exists essentially of DML domains solubilizing pheo- \\ phytin in a concentration of $15 \mathrm{~mol} \%$. During the DML main transition the pheophytin solubility \\ decreases to about $2 \mathrm{~mol} \%$, the excess pheophytin being precipitated within the surface layer. \\ During the pheophytin main transition an ordered structure below the surface layer is formed. \\ A stabilizing interaction between the pheophytin domains and their environment is observed and \\ discussed. \\ A sharp fluorescence change at a pressure below $0.5 \mathrm{dyn} / \mathrm{cm}$ indicates another phase transition. \\ It very probably involves an unwrapping of pheophytin from water molecules.
}

\section{Introduction}

The chloroplast thylakoid membrane contains the light harvesting chlorophyll molecules in a fairly large concentration (10 mol\%) [1]. However, in all artificial systems studied so far, concentration quenching of the excitation energy is extremely efficient even at lower concentrations $[2,3]$. On the other hand this process wasting the absorbed light energy is not effective in the photosynthetic unit. It is therefore conceivable that there is a specific interaction between the units forming the thylakoid membrane thus prohibiting concentration quenching. This interaction may be a chlorophyll-lipid binding [4], a chlorophyll-protein or a chlorophyll-water interaction. Specific arrangements arising from these bindings have been discussed by several authors [5-7].

In an effort to contribute to an understanding of these interactions we investigated monolayers and bilayer lipid vesicles containing the phospholipid $\alpha$-dimyristoyl-lecithin (DML) and chlorophylls. In a short note we provided evidence for the localization of chlorophyll in phospholipid vesicles [8]: The stereospecific quenching experiments suggested a localization of the porphyrin ring of chlorophyll a near the polar headgroup region, not protruding out into the water phase.

Reprint requests should be sent to Helmuth Heithier, Experimentelle Physik III, Universität Ulm, Oberer Eselsberg, D.7900 Ulm.
The present publication (part I) deals with optical and thermodynamical experiments on monolayers containing pheophytin a in variing relative amount. Part II will contain a comparative study on lipid bilayer vesicles [9]. It will show inhowfar the results obtained on monolayers can be trans. ferred to vesicles. Compared to previous studies on monolayers $[10,11]$ the present investigation exhibits mainly two new features:

(i) The pheophytin/lipid ratio was varied from zero to infinity in order to get information on the formation of chlorophyll domains and on the chlorophyll solubility in the lipid phase.

(ii) Surface pressure and fluorescence intensity were measured simultaneously as a function of area per molecule. By this the state of the monolayer could be characterized more accurately.

In special the experiments on mixed monolayers yield information on the following topics:

1. Phase separations are detected from pressure and from fluorescence data and an interaction between domains of the phases is observed and described within a model.

2. Using the pheophytin fluorescense as a probe different phase transitions of the monolayer are investigated and characterized.

3 . The pheophytin-pheophytin interaction is investigated from the pressure and concentration dependence of the fluorescence intensity.
This work has been digitalized and published in 2013 by Verlag Zeitschrift für Naturforschung in cooperation with the Max Planck Society for the Advancement of Science under a Creative Commons Attribution-NoDerivs 3.0 Germany License. 
4. The data obtained at high surface pressures indicate the formation of a specific pheophytin organization in the water subphase.

Especially the results on phase separations within the monolayer are important with respect to optical studies of in vivo systems. In these systems a phase separation is proposed too from a comparison of optical spectra and exciton calculations [13].

\section{Experimental}

Chlorophylls and lipids were prepared as described previously [8]. A circular Wilhelmy type film balance was built according to a draft of Fromherz [14a] and is similar to the one shown in ref. [14b]. It was embedded in an environmental chamber flushable with argon gas. The pressure could be measured to an accuracy of $\pm 0.2 \mathrm{dyn} / \mathrm{cm}$ and within that limit the pressure versus area curves of different runs were reproducible.

For the simultaneous recording of the pheophytin fluorescence a monochromator (Jobin-Yvon H 20) and a red sensitive photomultiplier were used [15]. Emission with the maximum at $680 \mathrm{~nm}$ was re- gistered. No shift of that maximum with pressure or temperature was observed within our experimental accuracy $(\approx 8 \mathrm{~nm})$. Excitation of the monolayer was achieved by a $200 \mathrm{~W}$ high pressure mercury lamp, intermitting suitable cut-off filters to excite only into the soret band region of pheophytin. The excitation beam was directed nearly parallel to the water surface, the emission beam was collected perpendicular to the surface.

The water used was demineralized and prepared with a filtering system (Millipore Mille $\mathrm{Q}^{\mathrm{TM}}$ ). The $\mathrm{pH}$ was maintained at 7.8 through a borax buffer. The film was prepared by first mixing $n$-hexane solutions of pheophytin and lipid in a syringe and then spreading the solution on the water surface. The layer was found to be unstable if the film was kept for more than a few minutes under a pressure below $0.5 \mathrm{dyn} / \mathrm{cm}$. Therefore immediately a film pressure of $10 \mathrm{dyn} / \mathrm{cm}$ was maintained for about 5 minutes. After evaporation of the solvent the film was then expanded and compressed. During the measurements the film remained stable as revealed by the reproducibility of pressure and fluorescence measurements.

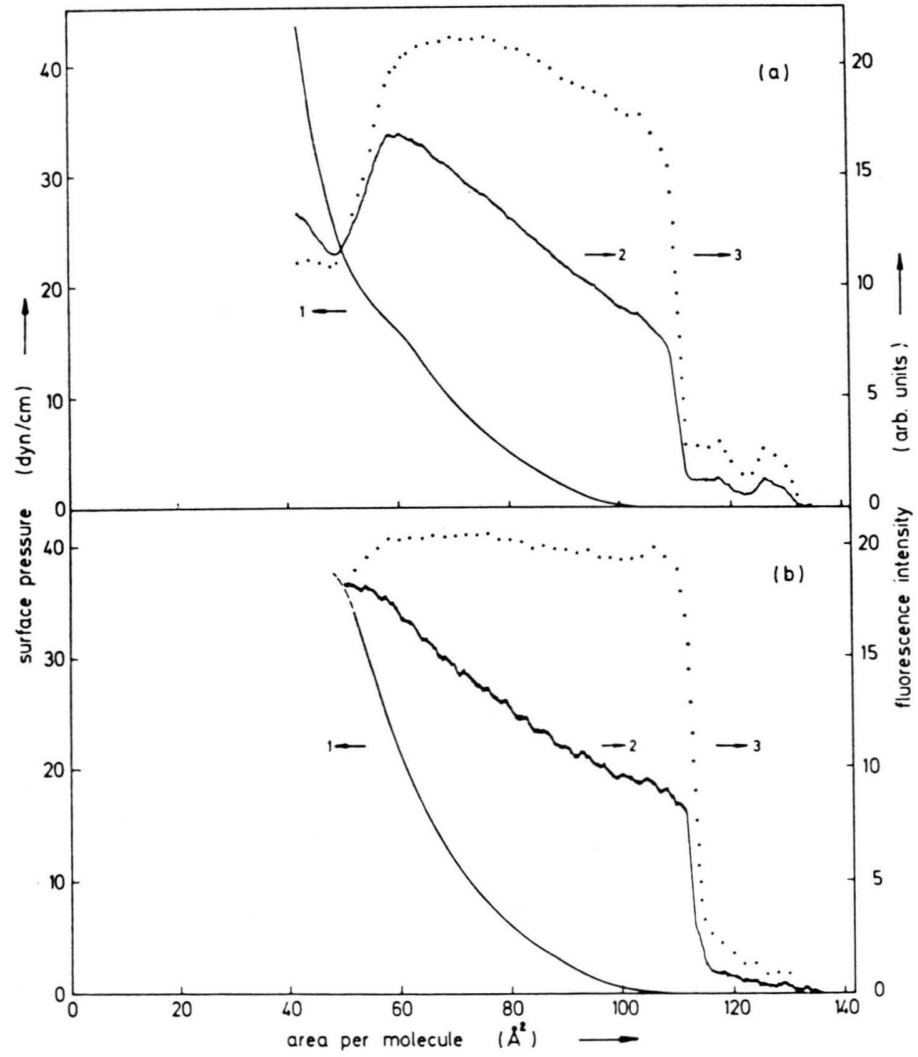

Fig. 1. Surface pressure (curves 1), fluorescence intensity (curves 2) and "normalized" fluorescence intensity (curves 3) as a function of area per molecule.

Fig. 1 a. Temperature $8{ }^{\circ} \mathrm{C}$, pheophytin concentration $X=4 \mathrm{~mol} \%$.

Fig. $1 \mathrm{~b}$ : Temperature $25^{\circ} \mathrm{C}, X=5 \mathrm{~mol} \%$. 


\section{Experimental Results}

In Fig. 1 the surface pressure versus area diagrams 1 are compared with the corresponding curves of the fluorescence intensity 2 . The monolayer consists of DML molecules containing a small amount of pheophytin molecules. The dotted curves 3 show the product fluorescence intensity $\mathrm{x}$ area/molecule. It

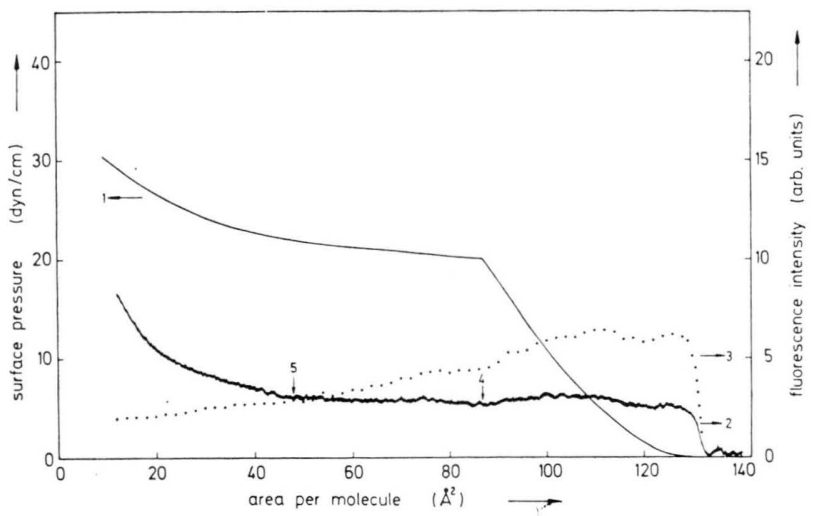

Fig. 2. Surface pressure (1), fluorescence intensity (2) and "normalized" fluorescence intensity (3) as a function of area per molecule for a pheophytin monolayer. Temperature $8^{\circ} \mathrm{C}$. yields kind of a "normalized" fluorescence intensity: it takes into account the linear increase of the optical density of the monolayer with the increase of the molecular density. This is a feature quite frequently met.

Curve 1 in Fig. 1 a shows the main transition of the monolayer beginning at $15.5 \mathrm{dyn} / \mathrm{cm}$. This transition, the solidification of the layer, is also observed as a decrease in the fluorescence intensity on elevating the pressure. The transition does not appear at high temperatures (Fig. $1 \mathrm{~b}$ ). Another prominent feature of all fluorescence versus area diagrams is the sharp fluorescence change at a pressure below $0.5 \mathrm{dyn} / \mathrm{cm}$. A possible explanation of this will be given in IV.6. The intensity change between these two transitions is due mainly to the change in optical density. This is proved by the nearly flat "normalized" curves 3 . The measurements on a pheophytin monolayer are given in Fig. 2. The pressure versus area diagram changes abruptly at an area of $87 \AA^{2} /$ molecule to become flat at smaller areas. The monolayer can be compressed as much as the apparatus allows, then expanded and compressed again with nearly the same

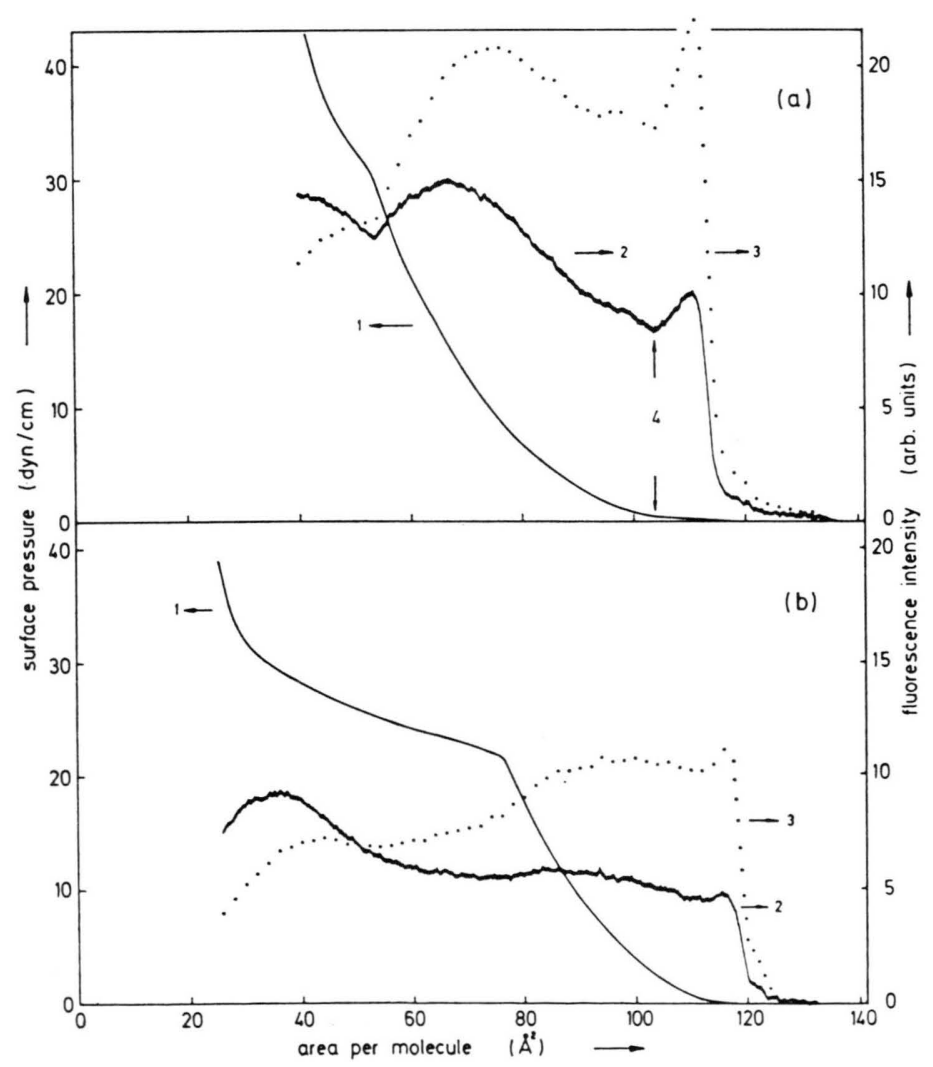

Fig. 3. Diagrams as in Figs 1 and 2 for an intermediate concentration range. Temperature $8{ }^{\circ} \mathrm{C}$.

Fig. 3 a: pheophytin concentration $X=20$ $\mathrm{mol} \%$.

Fig. $3 \mathrm{~b}: X=60 \mathrm{~mol} \%$. Arrow 4 indicates a change in the slopes of all diagrams in Fig. 3 a (c. f. IV.5). 


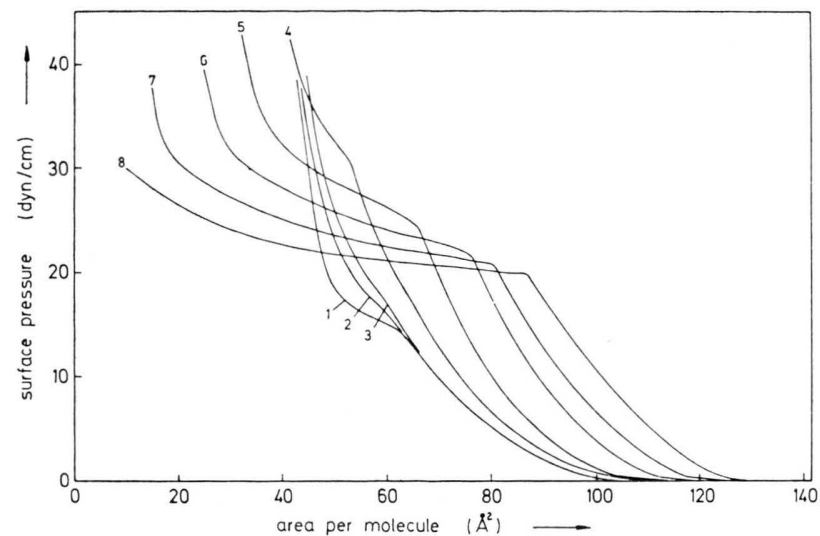

Fig. 4. Surface pressure versus area diagrams for DML monolayers containing pheophytin in different concentrations $X$. Temperature $8{ }^{\circ} \mathrm{C}$. (1) : $X=0 \mathrm{~mol} \%,(2): X=$ $4 \mathrm{~mol} \%$, (3) $X=10 \mathrm{~mol} \%$, (4) : $X=20 \mathrm{~mol} \%$, (5) : $X=$ $40 \mathrm{~mol} \%$, (6) : $X=60 \mathrm{~mol} \%$, (7) : $X=80 \mathrm{~mol} \%$, (8) $X=$ $100 \mathrm{~mol} \%$.

diagram. The monolayer transition is also observed as a slight change in the fluorescence intensity (arrow 4). Remarkable is also a second change in the slope of the fluorescence curve 2 (arrow 5), accompanied by a less pronounced change in the slope of the pressure versus area diagram. This change occurs at nearly twice the molecular density than the first change.

Whereas Figs 1 and 2 present data on monolayers containing DML or pheophytin in large excess, Fig. 3 gives examples for results obtained on monolayers containing both molecules in an appreciable amount. Changes in the slopes of the fluorescence are always accompanied by more or less pronounced changes in the slopes of the pressure versus area diagrams.

Fig. 4 summarizes the surface pressure versus area curves for DML monolayers containing pheophytin a in different concentrations. The curves can be classified into two distinct groups:

(A): Curves 1, 2 and 3 obtained for a pheophytin content between 0 and $10 \mathrm{~mol} \%$. For these layers the DML main transition is clearly observed, but it is broadened and its pressure is elevated with increasing pheophytin content.

(B): Curves 4 to 8 , corresponding to pheophytin concentrations between 20 and $100 \mathrm{~mol} \%$. For these solutions the sharp transition of the pure pheophytin monolayer is observed. With

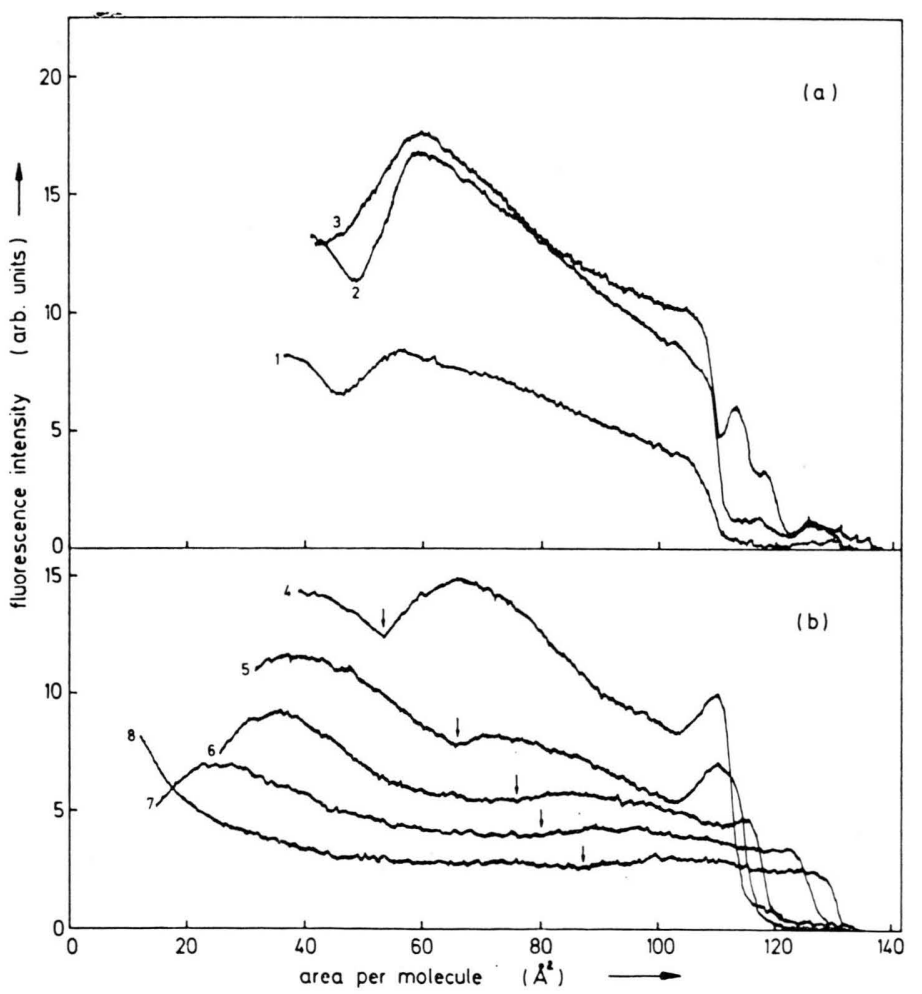

Fig. 5. Fluorescence intensity versus area for DML monolayers containing pheophytin in variing concentration $X$. (1) : $X=1 \mathrm{~mol} \%,(2): X=$ $4 \mathrm{~mol} \%, \quad(3): X=10 \mathrm{~mol} \%$, (4) : $X=20 \mathrm{~mol} \%$, (5) : $X=40 \mathrm{~mol} \%$, (6) : $X=60 \mathrm{~mol} \%,(7): X=$ $80 \mathrm{~mol} \%,(8): X=100 \mathrm{~mol} \%$. The arrows in Fig. $5 \mathrm{~b}$ indicate the area where the DML main transition in the surface pressure versus area diagrams in Fig. 4 is observed. 
decreasing pheophytin concentration the transition pressure $\pi_{\mathrm{K}}$ increases and the corresponding area $a_{\mathrm{K}}$ decreases.

An analogous classification can also be performed for the fluorescence curves in Fig. 5. Curves 1 to 3 in Fig. 5 a belong to group (A), showing the main transition of the DML monolayer. Curves 4 to 8 belong to class (B) showing the pheophytin transition indicated by arrows. Remarkable is also the cusp in the fluorescence at intermediate pheophytin concentrations $(20 \%, 40 \%, 60 \%)$ and at low pressure (smaller than $1 \mathrm{dyn} / \mathrm{cm}$ ).

\section{Discussion}

\section{IV.1. Phase separation in the liquid state}

A remarkable feature of the fluorescence is its increase with pheophytin concentration at a concentration below $10 \mathrm{~mol} \%$, but its decrease with further increasing pheophytin content. The latter is ascribed to concentration quenching [2]. In case of pheophytin this is expected to be effected by a reaction between an excited and an unexcited porphyrine ring [16]. As in the case of the well known pyrene excimere formation this leads to a quenching of the monomer fluorescence [17].

In order to evaluate the concentration dependence of the fluorescence intensity we used the data "normalized" intensity (curves 3 in Figs 1, 2 and 3 ) measured at a constant, but low pressure. The result is given in Fig. 6 for a pressure of $5 \mathrm{dyn} / \mathrm{cm}$. It is essentially identical between 2 and $12 \mathrm{dyn} / \mathrm{cm}$. The concentration dependence of the fluorescence intensity I in chlorophyll containing monolayers [16] and vesicles [4] is usually described by the empirical relation

$$
I=\frac{\operatorname{const} \cdot X}{1+a X^{2}}
$$

with $X=$ pheophytin concentration, $a=1 / X_{1 / 2}, X_{1 / 2}$ being the concentration where the quantum yield $I / X$ drops to half its value obtained at low concentration $X$. Eqn (1) yields a linear concentration dependence at small $X$ and a $1 / X$ dependence for large $X$, as given by the dashed curves in Fig. 6 . These curves have been obtained by a fit to the data obtained at pheophytin concentrations below $10 \%$ [15]. Curves a and c represent the error limits. The fit yields a value of $X_{1 / 2}=6.5 \pm 2 \mathrm{~mol} \%$, which is in

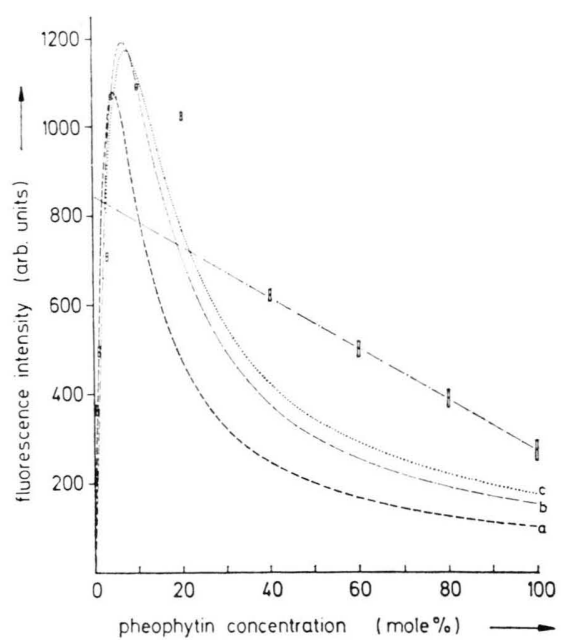

Fig. 6. "Normalized" fluorescence intensity as a function of pheophytin concentration at a constant surface pressure of $5 \mathrm{dyn} / \mathrm{cm}$. Temperature $8{ }^{\circ} \mathrm{C}$. Curves a, b and c correspond to a description according to Eqn (1), the straight line corresponds to a behaviour according to Eqn (2) (see IV.1).

the range of what has been published for chlorophylls in monolayers [16] and vesicles [4].

Obviously Eqn (1) is not applicable to the data obtained at large pheophytin concentration. Therefore we propose another model to explain the observed linear concentration dependence:

Within that model the monolayer is built up of two types of domains containing pheophytin in different concentrations. Domain 1 exists almost exclusively of pheophytin molecules, domain 2 is part of a DML monolayer containing pheophytin in a concentration $X_{0}$, its solubility limit. This value is constant for $X>X_{0}$, and in that case the fluorescence stems from both domain types $[I(1)$ and $I$ (2) ]. The fluorescence $I$ (total) of the whole monolayer can then be described by the following relation:

$$
I(\text { total })=\left(1-X^{\prime}\right) \cdot \mathrm{I}(2)+X^{\prime} \cdot I(1)
$$

with

$X^{\prime}=X-\left(X_{0} / 1-X_{0}\right) \cdot(1-X)$ and $X_{0} \leqq X \leqq 1$.

Eqn (2) yields the linear dependence of the fluorescence intensity observed for a pheophytin concentration larger than $40 \mathrm{~mol} \%$. The value of $X_{0}$ can be obtained from the intercept between curve $b$ in Fig. 6 and the straight line corresponding to Eqn (2). These curves describe correctly the low pheophytin concentration $\left(X \ll X_{0}\right)$ or the high 
concentration limit $\left(X \gg X_{0}\right)$, respectively. One obtains $X_{0}=15 \pm 5 \mathrm{~mol} \%$.

Besides the observed concentration dependence there are five more arguments in favour of a separation of the monolayer into the two kinds of domains just described:

1. The surface pressure versus area isotherms (Fig. 4) can be classified into two distinct groups. Group (A), observed for a pheophytin concentration below $10 \%$ and group (B) observed for a pheophytin concentration larger than $20 \%$.

2. The same classification can be performed with the fluorescence versus area isotherms (Fig. 5).

3. Fig. 7 a shows the area per molecule for a low, but constant pressure. The deviation of the experimental points from a straight line linking the points for $X=0$ and $X=1$ indicates that the solution is not ideal. The behaviour indicated by the two straight lines in Fig. 7 a would be obtained if two types of noninteracting domains were present. The data do fit these lines, but we are aware of the additional complications inferred by the interaction between the domains that we observe.

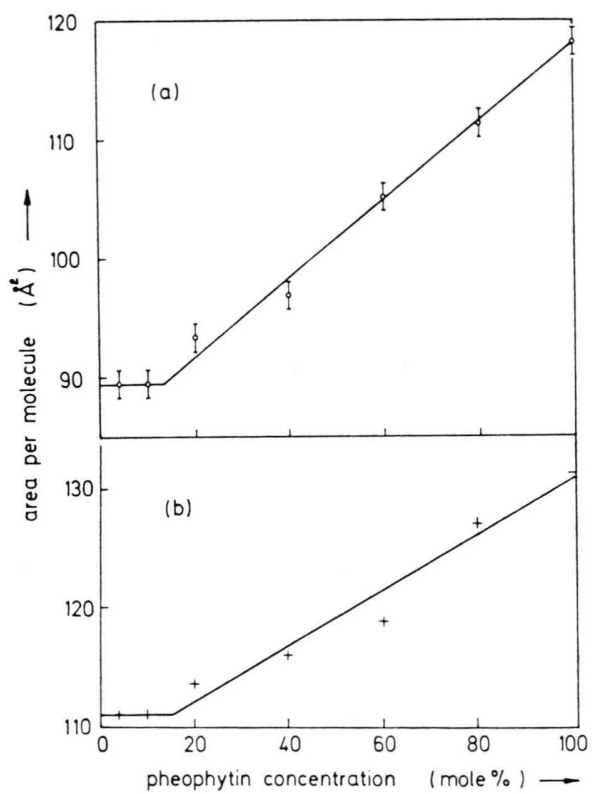

Fig. 7. a) : Area per molecule for a surface pressure of $2 \mathrm{dyn} / \mathrm{cm}$. b): Area per molecule corresponding to the onset of the phase transition at low pressures. This is defined for convenience as the point where the fluorescence performs half its change during the transition (c.f. Fig. 5).
4. We can extend the model to describe the interaction between the domains (see IV.5). This interaction is proved and evaluated essentially from the concentration dependence of the pressure at which the phase transition of the pheophytin domains is observed (c.f. Fig. 4).

5. We will show that the pheophytin transition involves a deviation of the molecules into the subphase. Therefore at a pressure above $40 \mathrm{dyn} /$ $\mathrm{cm}$ the pheophytin domains should not contribute to the monolayer area. This is definitely observed and discussed in section IV.3.

\section{IV.2. Main transition of the DML domains}

Having established the existence of two different types of domains in the mixed monolayer we will now turn to a description of the main phase transitions of the domains. An extended thermodynamical study of phase transitions in lecithin monolayers has been performed only recently [18]. Therefore we will focus on the change in pheophytin arrangement during the transition. This is one of the topics of this work.

The DML main transition is clearly observed from the "pressure"-but more prominently from the "fluorescence versus area diagram" (Fig. 1 a). Thus the pheophytin fluorescence presents an excellent probe for a characterization of this transition. We do not want to stress that point since we yet measured systematically only at a temperature of $8{ }^{\circ} \mathrm{C}$, i.e. a temperature where the lipid main transition is clearly observed. The curves in Fig. $1 \mathrm{~b}$, measured at high temperatures are merely a check that the fluorescence change really reflects the lipid main transition. What is the reason for the fluorescence decrease (by about a factor of 2 in Fig. 1 a) on solidification of the DML monolayer? The following explanations may be taken into account:

(1) Single pheophytin molecules may closer approach the water phase that quenches its fluorescence. A fluorescence decrease independent of pheophytin concentration would result. This is clearly not observed: At low pheophytin concentration the fluorescence decrease is much less pronounced than at a larger pheophytin concentration (curve 1 compared to curve 2 in Fig. 5 a). This concentration dependence demonstrates that the fluorescence decrease is due to a cooperation of pheophytin molecules. 
(2) During the transition domains of pheophytin molecules may be formed and then pushed into the water phase where they don't fluorescence. This is not very probable, since we will show that in case of a pheophytin concentration below $20 \mathrm{~mol} \%$ a pressure at least larger than $30 \mathrm{dyn} / \mathrm{cm}$ is needed to segregate pheophytin out into the subphase. In fact, it turns out that the presence of DML molecules tends to stabilize pheophytin domains within the monolayer surface (c.f. IV.5).

(3) Instead we do favour another model for the solid containing pheophytin in a concentration below $10 \mathrm{~mol} \%$ : Pheophytin is segregated out within the monomolecular film. Regions of extremely large pheophytin concentrations are formed where an efficient self quenching occurs. We do not want to speculate too much about a more detailed model of the solid lipid phase. We merely want to mention that our experimental data can be explained by a solid lipid phase containing pheophytin in a concentration of at most $2 \mathrm{~mol} \%$. The excess pheophytin forms domains that are pushed into the subphase only at much larger pressures and at total pheophytin concentrations exceeding $10 \mathrm{~mol} \%$.

\section{IV.3. Main transition within the pheophytin domains}

Fig. 2 shows the experimental facts concerning the pure pheophytin monolayer. It is important to note that the layer does not really collapse at a pressure larger than $\pi_{\mathrm{K}}=20 \mathrm{dyn} / \mathrm{cm}$. Curve 1 is quite reversible, reproducible and does not show any discontinuities as to be expected for an unstable surface layer. We will now show that during the main transition pheophytin is segregated into the subface forming another ordered structure still interacting with the surface layer.

The deviation of pheophytin molecules into the subphase is proved by the following findings:

(1) At a pressure of $30 \mathrm{dyn} / \mathrm{cm}$ the area per molecule of the pure pheophytin layer amounts to less than $10 \AA^{2}$ (curve 1 in Fig. 2). This is much less than the area needed by a pheophytin molecule.

(2) Fig. 8 gives the area per molecule as a function of concentration at a pressure of $40 \mathrm{dyn} /$ $\mathrm{cm}$. The measured points do not deviate too

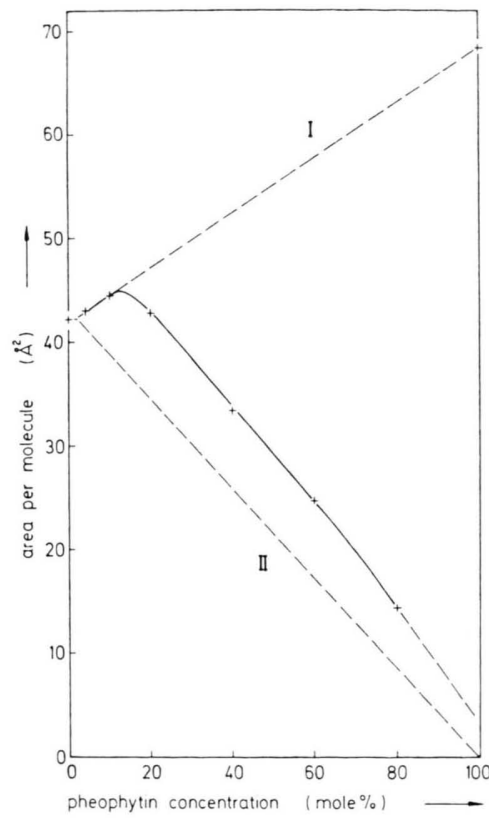

Fig. 8. Area per molecule for a surface pressure of 40 dyn $/ \mathrm{cm}$. Line II gives the area needed by the solidified DML domains containing $2 \mathrm{~mol} \%$ pheophytin. The behaviour according to line I would be observed if also the pheophytin domains would remain in the surface layer. The area needed by these domains has been calculated from an extrapolation of curve 1 in Fig. 2 to higher pressures.

much from curve II. This shows that the area is essentially determined by the fraction of DML molecules within the monolayer.

(3) During the phase transition of the monolayer the slope in the fluorescence versus area curve changes slightly (arrow 4 in Fig. 2), but then does not rise with increasing pressure. This has to be expected for a monolayer the optical density of which increases with the molecular density. This indicates that $e$.g. on doubling the molecular density between arrows 4 and 5 in Fig. 2 the density of fluorescing molecules within the surface layer remains unchanged *.

(4) The absorption spectrum measured by Tweet et al. [10] for their so-called unstable region shows an additional red-shifted band. This has to be expected for aggregated pheophytin molecules in the water phase.

* The same conclusion may be drawn from the "corrected fluorescence" curve 3 in Fig. 2 . We should, however, mention that in case where the optical density of the fluorescent monolayer is not inversely proportional to the measured area per molecule, this curve does not really represent a correction. 
It is yet speculative what kind of an ordered structure pheophytin assumes in the water phase. It is, however, interesting that the slopes of the pressure and fluorescence curves change at nearly half the area at which the phase transition occurs (compare arrows 4 and 5 in Fig. 2). This may indicate that a second (nonfluorescing) monolayer is formed below the surface layer. It is, however, also possible that during the phase transition pheophytin micelles are formed remaining close to the surface layer or that the monolayer spontaneously bends and forms ripples as has been observed from electron micrographs in lecithin vesicles [19].

\section{IV.4. Interaction between a pheophytin domain and its environment}

The following section is intended to establish an interaction between a pheophytin domain and its environment. In our understanding a domain exists of a regular arrangement of molecules, hence the environment is composed of differently arranged molecules. It turns out that our description of the domain interaction holds even for very low DML concentrations. This means that with respect to the interaction there is no difference which ever molecule composes a domain's environment. This indicates that the interactions are dominated by steric forces. Within our model the concentration dependence of these interactions is inferred by a composition dependence of the domain size.

Qualitatively the interaction is manifested in the following experimental findings:

(i) At a pressure of $40 \mathrm{dyn} / \mathrm{cm}$ the area per molecule increases with pheophytin concentration in the concentration range between $2 \mathrm{~mol} \%$ and $10 \mathrm{~mol} \%$ (Fig. 8). This proves that the monolayer contains pheophytin in a concentration exceeding its solubility ( $2 \mathrm{~mol} \%$, see IV.2) in the solid DML phase. Thus there are precipitations of pheophytin domains which, even at such a high pressure, do not segregate into the water phase. There is obviously an interaction within the monolayer stabilizing the pheophytin domains.

(ii) The pressure $\pi_{\mathrm{k}}$ at which the main transition of pheophytin occurs increases with decreasing pheophytin content. This also indicates a stabilizing interaction between a domain and its environment and is evaluated within a model more quantitatively in a future publication [20].

This model has been used to interprete phase separations in bilayer lipid vesicles [21, 22]. Extended to the present problem the monolayer contains pheophytin domains, the number of which is fixed in a concentration range between $20 \mathrm{~mol} \%$ and 100 mol\%. Hence the domain size decreases with decreasing pheophytin concentration. Therefore the number of molecules near the domain walls increases relative to the number of molecules in the bulk. This causes an increased interaction between a domain and its environment and is reflected in the concentration dependence of $\pi_{\mathrm{k}}$ : Since the interaction stabilizes the domain at lower pheophytin concentration a larger pressure $\pi_{\mathrm{k}}$ is needed to enforce the phase transition.

\section{IV.5. The transition at low pressures $(>0.5 \mathrm{dyn} / \mathrm{cm})$}

The fluorescence versus area curves in Figs 1, 2, 3 and 5 show a sharp fluorescence increase at very low pressures. It is yet highly speculative to give a physical interpretation of the change of the state of the monolayer. Still we would like to present some experimental facts:

(1) Fig. $7 \mathrm{~b}$ gives the composition dependence of the area per molecule at which the fluorescence intensity performs half of its change observed during the phase transition. Obviously this curve resembles that of Fig. 7 a obtained for a pressure of $2 \mathrm{dyn} / \mathrm{cm}$. This indicates that, irrespective of the pheophytin concentration the transition occurs at the same pressure.

(2) At a higher temperature the onset of the transition appears at a slightly larger area per molecule, unlike the liquefaction of a van der Waals gas (c.f. Fig. 1). This indicates the envolvement of a large entropy change and a more ordered structure in the expanded state.

(3) The sharp fluorescence change suggests that the distance between the porphyrin ring and the fluorescence quenching water molecules is drastically changed.

(4) In an intermediate concentration range one observes a cusp in the fluorescence intensity and also a change in the slope of the pressure versus area curves (c.f. arrow 4 in Fig. 3 a). 
This indicates that the transition has a certain width (from $105 \AA^{2} /$ molec. to $115 \AA^{2} /$ molec.). On increasing the density the pheophytin concentration in the DML domains increases. This causes a fluorescence increase and then a decrease due to self-quenching at larger pheophytin concentrations as is quantitatively given in Fig. 6.

These facts suggest that during the transition pheophytin is dissolved in the lecithin part of the monolayer and is freed from water molecules that were previously arranged around it. Such an entropic transition has recently been observed with monolayers of the hydrophobic molecule PBLG (poly- $\gamma$ benzyl-L-glutamate) [23]. More temperature dependent experiments are intended to further elucidate this point.

\section{Concluding Remarks}

The investigation of a phase separation that we have observed on a monolayer of pheophytin and DML may have considerable impact on photosynthesis research. It is highly probable that phase separations exist in the photosynthetic unit, too, as has been discussed by several authors (see $e$. g. refs. $[3,4,6])$.

The functioning of the photosynthetic system is also highly dependent on the kind of arrangement of the chlorophylls [24]. Unfortunately, the presented experiments do not decide whether the pheophytin molecules form linear arrays. This has been proposed for chlorophyll in vivo by Shipman [13]. It is, however, important to note that our model to explain the domain interaction considers twodimen-

[1] K. Sauer, Bioenergetics of photosynthesis, ed. Govindjee, Academic Press, New York 1975.

[2] W. E. Watson and R. Livingston, J. Chem. Phys. 18, 802 (1950).

[3] G. Porter, Naturwissenschaften 63, 207 (1976).

[4] G. S. Beddard, S. E. Carlin, and G. Porter, Chem. Phys. Lett. 43, 27 (1976).

[5] K. H. Ballschmiter and J. J. Katz, J. Am. Chem. Soc. 91, 2661 (1969).

[6] J. J. Katz, W. Oettmeier, and J. R. Norris, Phil. Trans. Roy. Soc. London, Sev. B 273, 227 (1976).

[7] F. K. Fong, Appl. Phys. 6, 151 (1975).

[8] J. Luisetti, H. Möhwald, and H.-J. Galla, Biochem. Biophys. Res. Commun. 78, 754 (1977).

[9] J. Luisetti, H.-J. Galla, and H. Möhwald, to be published. sional domains. The experimental results are not consistent with a onedimensional domain model.

We also performed some preliminary experiments on chlorophyll b containing monolayers in order to decide inhowfar the pheophytin results can be transferred to chlorophyll [15]. We obtained evidence for a phase separation, a dimerization and a chlorophyll b arrangement different from that of pheophytin a. Still, the results are much less conclusive predominantly since (i) the monolayer fluorescence was too small to be observed for chlorophyll concentrations larger than $30 \mathrm{~mol} \%$ and (ii) the phase transition corresponding to the main transition of the pheophytin domains could not be observed in the pressure versus area diagrams.

This shows that the pheophytin results can be transferred to chlorophyll only to a very limited extent. The experiments will therefore have to be performed with each type of photosynthetically active molecules. The presented results hopefully demonstrated that this will contribute to a better understanding of the intermolecular interactions and the arrangement of these molecules.

Part II of this series of publications will report on experiments on DML vesicles containing chlorophylls. It will also answer the question inhowfar the results on monolayers can be transferred to vesicles. Presently we should already mention that a phase separation on a vesicle also explains the nonexponentiallity of the fluorescence decay. This has been observed by Porter and Co-workers [4].

We profited from critical and informative discussions with Prof. E. Sackmann and Prof. H. Gruler. The technical assistance of H. Kuttruff, U. Theilen and H. Maute is greatfully acknowledged.

[10] W. D. Bellamy, G. L. Gaines, and A. G. Tweet, J. Chem. Phys. 39, 2528 (1963). - A. G. Tweet, G. L. Gaines, and W. D. Bellamy, J. Chem. Phys. 40, 2596 (1964). - A. G. Tweet, W. D. Bellamy, and G. L. Gaines, J. Chem. Phys. 41, 2068 (1964).

[11] T. Trosper, R. B. Park, and K. Sauer, Photochem. Photobiol. 7, 451 (1968).

[12] W. Sperling and B. Ke, Photochem. Photobiol. 5, 857 (1966).

[13] L. L. Shipman, J. Phys. Chem. 81, 2180 (1977).

[14] a) P. Fromherz, Rev. Sci. Instr. 46, 1380 (1975). b) H. Kuhn, D. Möbius, and H. Bücher, Techniques of Chemistry (Eds. A. Weissberger and B. W. Rossiter), Vol. 1, Part IIIB, pp. 577-702, Wiley, New York 1972. 
[15] H. Heithier, Diplomarbeit, Universität Ulm, 1978.

[16] S. M. de B. Costa, J. R. Froines, J. M. Harris, R. M. Leblanc, B. H. Orger, and G. Porter, Proc. Roy. Soc. London A 326, 503 (1972).

[17] Th. Förster, Angew. Chemie 81, 364 (1969).

[18] O. Albrecht, H. Gruler, and E. Sackmann, J. de Physique 39, 301 (1978).

[19] C. Gebhardt, H. Gruler, and E. Sackmann, Z. Naturforsch. 32 c, 581 (1977).
[20] H. Heithier, H.-J. Galla, and H. Möhwald, to be published.

[21] E. Sackmann and H. Träuble, J. Amer. Chem. Soc. 94, 4482, 4492 (1972). - H. Träuble and E. Sackmann, J. Amer. Chem. Soc. 94, 4499 (1972).

[22] H.-J. Galla and E. Sackmann, Biochim. Biophys. Acta 401, 509 (1975).

[23] P. Gebhardt, O. Albrecht, and H. Gruler, private communication.

[24] H. T. Witt, Naturwissenschaften 63, 23 (1976). 